\title{
Improvement of chatter stability in boring operations with passive vibration absorbers
}

\author{
M.H. Miguélez ${ }^{\text {a }}$, L. Rubio ${ }^{\text {a }}$, J.A. Loya ${ }^{\text {b }}$, J. Fernández-Sáez ${ }^{\text {b,* }}$ \\ ${ }^{a}$ Department of Mechanical Engineering, University Carlos III of Madrid, Avda. de la Universidad, 30, 28911 Leganés, Madrid, Spain \\ ${ }^{\mathrm{b}}$ Department of Continuum Mechanics and Structural Analysis, University Carlos III of Madrid, Avda. de la Universidad, 30,28911 Leganés, Madrid, Spain
}

Keywords:

Stability

Chatter

Passive absorbers

Boring bar

Analytical approach
This paper is focused on the behavior of boring bars with a passive dynamic vibration absorber (DVA) for chatter suppression. The boring bar was modeled as a cantilever Euler-Bernoulli beam and only its first mode of vibration was considered. The stability of the two-degree-of-freedom model was analyzed constructing the stability diagram, dependent on the bar characteristics and on the absorber parameters (mass, stiffness, damping, and position). Two analytical approaches for tuning the absorber parameters were compared. The selection criterion consisted on the maximization of the minimum values of the stability-lobes diagram. Subsequent analysis performed in this work, allowed formulating of new analytical expressions for the tuning frequency improving the behavior of the system against chatter.

\section{Introduction}

Machining is one of the most common processes for material removal in industry. Cutting operations such as turning, milling, boring, and grinding, are sometimes associated with degrading vibrations resulting in poor surface quality, decreased removal rate and accelerated tool wear [1]. Chatter or self-excited vibration is the most significant type of vibration in machining operations. Regeneration and mode coupling are the main phenomena leading to chatter, the former being the more detrimental for machining operations.

Some works on chatter analysis are briefly summarized below.

Although Taylor [2] had identified the problem of chatter for machining productivity at the beginning of the 20th century, the earliest study of chatter theory in simple machine-tool systems was stated by Arnold [3] in the 1940s based on experimental turning tests, investigating chatter under controlled conditions.

Tobias and Fishwick [4] proposed a single-degree-of-freedom model, identifying the regeneration of chip thickness and modal coupling as the main mechanisms leading to the onset of chatter vibrations.

Later, Tlustly and Polacek [5] and Merritt [6] worked on the stability conditions in machine tools, identifying structural dynamics of the machine and the subsequent cuts on the machined surface as significant sources of chatter. Merritt [6]

\footnotetext{
* Corresponding author. Tel.: +341 624 9964; fax: +3416249430.

E-mail address: ppfer@ing.uc3m.es (J. Fernández-Sáez).
}

proposed a simple stability criterion and discussed the stability of structures with $\mathrm{N}$-degrees of freedom. Also, Tobias [7] reviewed extensively the state of the art concerning the dynamic behavior of the lathe and chatter theory. The analysis of chatter phenomena was improved considering the chip-thickness variation and the phase lag of the undulation of the surface.

In the 1970 s in a reference work, Hanna and Tobias [8] proposed a single-degree-of-freedom time-delay differential equation including non-linear terms, due to structural stiffness and cutting force.

$\mathrm{Wu}$ and Liu $[9,10]$ derived a mathematical model taking into account the effect of dry friction at the tool-chip interface, which was identified as a source of chatter due to its velocity-dependent nature.

In the 1990s Budak and Altintas [11,12] developed several works for milling, modeling the cutting tool as a two-degree-offreedom linear system. The instantaneous chip thickness, tool geometry, and tooth number were considered for the estimation of the cutting force. Recently, similar models have been used for chatter predictions in milling [13,14]. Regenerative chatter stability of milling processes has also been investigated using a fuzzy algorithm [15] in order to consider uncertainty or variability in the model input parameters.

Concerning turning operations, a multiple-degree-of-freedom model for chatter prediction, based on compliance between the cutting tool and the workpiece was presented in [16]. The works focusing on chatter have been steadily increasing in recent years.

The problem of vibration becomes more significant when a flexible tool is used, as in the case of internal turning operations. 
Boring operations need long and slender bars to machine the internal zones of the workpiece (see Fig. 1a). Geometrical requirements of the tool are related to degrading vibrations, influencing not only surface quality, but also tool durability and productivity. Vibrations also have environmental consequences due to the high noise levels produced. The interest of these processes in industry and the special geometry of the tool have motivated the development of numerous works investigating chatter in boring operations.

The stability behavior of a slender boring bar was studied by Parker [17]. The boring bar was modeled as a two-degree-offreedom mass-spring-damper system. The mode coupling was experimentally analyzed for a range of cutting parameters. The analysis of boring-bar vibrations is usually based on the lowerorder bending modes of the clamped boring bar [18], although the clamping conditions of the bar also influences its dynamic properties [19]. Zhang and Kapoor [20] developed a two-degreeof-freedom model of a clamped boring bar with four cutting-force components. Andrén et al. [21] compared an analytical Euler-Bernoulli model with a time-series approach to investigate boring-bar chatter.

Not only characteristics of the boring bar such as clamping conditions have been analyzed. Different authors [22-27] have also focused attention on the geometrical details of the insert influencing the cutting force and the dynamic behaviour of the boring bar.

Different strategies have been developed to avoid or diminish vibrations in boring operations. Improved tool holder and clamping design [19] have demonstrated the ability to improve the dynamic behavior of the system. On the other hand, continuous improvement has been achieved in the chatter control of boring, including sophisticated methods, such as the use of electro-rheological [28] and magneto-rheological fluids [29] and active dynamic absorbers [30-33]. However, the use of passive dynamic absorbers [34-39] is a simple solution and it is still a promising field of research for chatter suppression, not only in boring operations.

The stability of boring bars with a passive dynamic absorber is commonly analyzed by modeling the bar as an Euler-Bernoulli beam, with two degrees of freedom. The first degree of freedom corresponds to the first mode of vibration of the bar, and the second is associated with the dynamic absorber, based on an elastic spring and a linear damper. Designing the absorber, involves the identification of the mass, the stiffness and the damping optimizing the dynamic response of the bar.

Rivin and Kang [34] presented an analytical approach to design an absorber and their detailed and comprehensive experimental study demonstrated significant performance improvements using their design procedure. Tarng et al. [35] manually tuned a vibration absorber and were able to modify the frequency response function, FRF, of the cutting tool. Both theoretical and experimental results showed the improvement of cutting stability. Ema and Marui [36] carried out bending, impact, and cutting tests investigating improvements in the damping capability of boring tools and suppression of chatter vibration using impact dampers. Lee [37] demonstrated that the dynamic response of the cutting tool was improved due to the presence of a passive absorber when the natural frequency of the dynamic vibration absorber was close to the natural frequency of the cutting tool and the absorber had a large damping ratio. Sims [38] proposed an analytical method relevant for a wide range of machining-chatter problems. In turning and boring operations, passive vibration absorbers can be tuned using the analytical technique developed in [38]. Moradi et al. [39] analyzed the influence of the absorber position along the boring bar on vibration response. The absorber parameters were selected minimizing the free-end deflection of the boring bar, however, the damping of the absorber was neglected in this approach. Recently, Saffury and Altus [40] proposed the use of viscoelastic bars as an alternative to a dynamic vibration absorber.

\section{a}

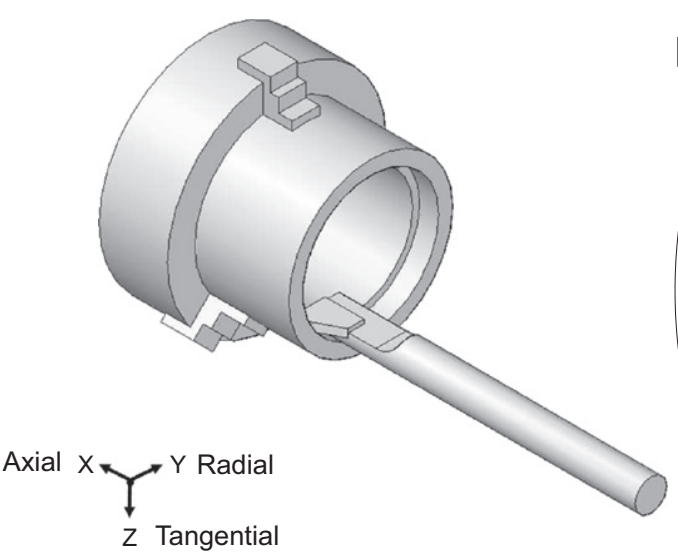

b
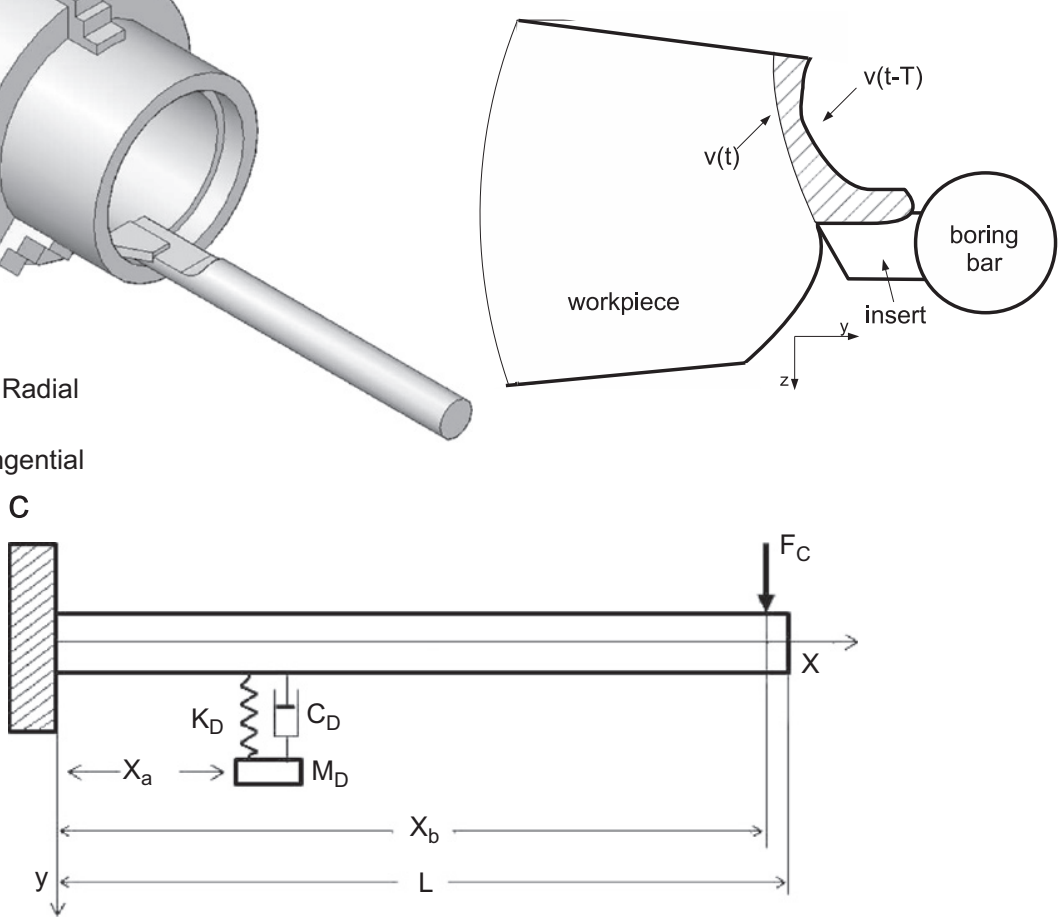

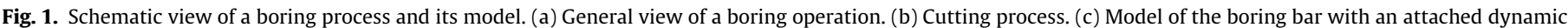
vibration absorber (DVA). 
This paper is focused on the stability of a boring bar with a passive dynamic absorber located at a generic section of the tool holder. The boring bar was modeled as a cantilever Euler-Bernoulli beam and only its first mode of vibration was taken into account. The stability of the two-degree-of-freedom model was analyzed in terms of the stability diagram dependent on the bar characteristics and also on the absorber parameters (mass, stiffness, damping, and position). Both the classical method introduced by Den Hartog [41] — considering the modulus of the FRF of the system - and the method recently proposed by Sims [38] — considering real part of the FRF-were used for tuning the absorber parameters. The selection criterion consisted of the maximization of the minimum values of the stability-lobes diagram. Subsequent local analysis, allowed us to formulate simple analytical expressions for the optimal tuning frequency. These new analytical expressions have been compared with those proposed by Den Hartog [41] and Sims [38].

The paper has been structured in seven sections. The aim of the paper is introduced in Section 1, while the problem formulation and the statement of motion equations are presented in Section 2. The analysis of system stability and the stability-diagram construction are summarized in Section 3. Comparison between both analytical methods of Den Hartog [41] and Sims [38] is shown in Section 4, discussing the ability of both approaches to help in the absorber design. In Section 5, new analytical expressions for the optimal tuning frequency are presented. The capability of the new method is shown in Section 6 with a practical example, and finally conclusions are presented in Section 7. Some mathematical details of calculation are included in the Appendix.

\section{Model of a boring bar with a vibration absorber}

A schematic view of a boring operation is illustrated in Fig. 1a. Boring processes involve the use of slender bars (tool) more flexible than the workpiece. The bar is much more rigid along the feed or axial direction than along the radial and tangential bending directions. On the other hand, bar stiffness is higher under torsion than under bending solicitations. Hence, bending vibrations in both radial and tangential directions should be accounted. However, geometrical analysis of the tool has shown that deflections in tangential direction ( $z$ axis in Fig. 1a) can be considered negligible in chip thickness variation [24].

Thus, the boring tool can be modeled as a uniform Euler-Bernoulli beam of length $L$, cross-sectional area $A$, Young's modulus $E$, density $\rho$, and damping $c$, with one end clamped and the other free, vibrating in radial direction $(x-y$ plane in Fig. 1a).

At a certain section located at distance $x_{b}$ from the clamped end, the cutting force, which is considered to be proportional to the cross-sectional area of the cut, is applied.

A simple formulation for the estimation of the chip section was assumed. Geometrical details of the tool, such as the insert nose radius, were not accounted in the model. Thus, just the variation of the depth of cut, resulting from surface undulations due to the previous cutting pass of the tool, was considered for the estimation of the radial component of cutting force (see Fig. 1b).

Then, in accordance with the classical regenerative chatter theory (see [7], for instance), the radial component of cutting force, $F_{y}$, relevant to analyze the regenerative process is given by

$F_{y}=k_{c} w_{c}(v(x, t-T)-v(x, t)) \delta\left(x-x_{b}\right)$

where $k_{c}$ is a constant parameter depending on both the specific cutting force and the cutting angles of the tool, $w_{c}$ is the chip width, $v(x, t)$ is the dynamic transverse displacement of the boring bar, and $T=2 \pi / \Omega$ is the delay between the current time and previous time at which the tool has passed the point under consideration, $\Omega$ being the spindle rotational speed and $\delta$ the Dirac delta function.

Despite its simplicity, this approach has been widely used to analyze the dynamic behavior of the boring bar [29,32,39].

The beam has a passive dynamic absorber (DVA) attached at a section located at distance $x_{a}$ from the clamped end (see Fig. 1c). The dynamic absorber is characterized by a free mass $M_{D}$, equivalent spring constant $K_{D}$ and damping constant $C_{D}$.

With these assumptions, the transverse displacement of the boring bar $v(x, t)$ and the displacement of the mass absorber, $V_{D}$, can be determined as the solutions of the following equations:

$$
\begin{aligned}
& \rho A \frac{\partial^{2} v(x, t)}{\partial t^{2}}+c \frac{\partial v(x, t)}{\partial t}+E I \frac{\partial^{4} v(x, t)}{\partial x^{4}} \\
& \quad+\left[C_{D}\left(\frac{\partial v(x, t)}{\partial t}-\frac{d V_{D}(t)}{d t}\right)+K_{D}\left(v(x, t)-V_{D}(t)\right)\right] \delta\left(x-x_{a}\right) \\
& \quad+k_{c} w_{c}[v(x, t)-v(x, t-T)] \delta\left(x-x_{b}\right)=0 \\
& M_{D} \frac{d^{2} V_{D}(t)}{d t^{2}}+C_{D}\left[\frac{d V_{D}(t)}{d t}-\left.\frac{\partial v(x, t)}{\partial t}\right|_{x=x_{a}}\right]+K_{D}\left[V_{D}(t)-v(x, t)\right]=0
\end{aligned}
$$

In the following, it is assumed that the boring-bar deflection at any point $x$ can be expressed as

$v(x, t)=L \sum_{j} q_{j}(t) \phi_{j}(x)$

where $q_{i}(t)$ represent the unknown time-dependent generalized coordinates and $\phi_{j}(x)$ are the orthogonal eigenfunctions of the clamped-free beam without DVA, given by

$\phi_{j}(x)=\cosh \lambda_{j} x-\cos \lambda_{j} x-\frac{\cosh \lambda_{j}+\cos \lambda_{j}}{\sinh \lambda_{j}+\sin \lambda_{j}}\left(\sinh \lambda_{j} x-\sin \lambda_{j} x\right)$

where the eigenvalues $\lambda_{j}$ are the solutions of the transcendental equation:

$\cosh \lambda_{j} x \cos \lambda_{j} x+1=0$

The first three values of $\lambda_{j}$ that satisfy this equation are

$\lambda_{1}=1.8751, \quad \lambda_{2}=4.6941, \quad \lambda_{3}=7.8548$

Substituting Eq. (4) into Eqs. (2) and (3), and using the new non-dimensional variables:

$\tilde{x}=\frac{x}{L}, \quad a=\frac{x_{a}}{L}, \quad b=\frac{x_{b}}{L}, \quad \tilde{V}_{D}=\frac{V_{D}}{L}$

$\tilde{t}=\omega_{0} t, \quad \tilde{T}=\omega_{0} T, \quad \omega_{0}=\sqrt{\frac{E I}{\rho A L^{4}}}$

Eqs. (2) and (3) can be rewritten omitting tildes for simplicity:

$$
\begin{aligned}
& \rho A L \omega_{0}^{2} \sum_{j} \phi_{j} \ddot{q}_{j}+c L \omega_{0} \sum_{j} \phi_{j} \dot{q}_{j}+\frac{E I}{L^{3}} \sum_{j} \phi_{j}^{I V} q_{j}(t) \\
& +\left[\frac{C_{D} L \omega_{0}}{L}\left(\sum_{j} \phi_{j} \dot{q}_{j}-\dot{V}_{D}\right)+\frac{K_{D} L}{L}\left(\sum_{j} \phi_{j} q_{j}(t)-V_{D}\right)\right] \delta(\bar{x}-a) \\
& +\frac{k_{c} w_{c} L}{L}\left[\sum_{j}\left(q_{j}(t)-q_{j}(t-T)\right)\right] \delta(\bar{x}-b)=0
\end{aligned}
$$

$M_{D} L \omega_{0}^{2} \ddot{V}_{d}+C_{D} L \omega_{0}\left[\dot{V}_{D}-\sum_{j} \phi_{j}(a) \dot{q}_{j}(t)\right]+K_{D} L\left[V_{D}-\sum_{j} \phi_{j}(a) q_{j}(t)\right]=0$

In the above equation, $(\bullet)$ indicates temporal derivatives and $\phi_{j}^{I V}$ represents the fourth order derivative with respect to the non-dimensional spatial variable $\bar{x}$. 
Multiplying both sides of Eq. (8) by the $k_{t h}$ eigenfunction $\phi_{k}(x)$ and integrating over the non-dimensional beam length, we obtain the following set of delay differential equations:

$$
\begin{aligned}
& \ddot{q}_{k}(t)+2 \zeta_{k} \lambda_{k}^{2} \dot{q}_{k}(t)+\lambda_{k}^{4} \xi_{D} \phi_{k}(a)\left(\sum_{j} \phi_{j}(a) \dot{q}_{j}(t)-\dot{V}_{D}\right) \\
& +\lambda_{D}^{4} \phi_{k}(a)\left(\sum_{j} \phi_{j}(a) q_{j}(t)-V_{D}\right)+k_{r} \phi_{j}(b) \sum_{j} \phi_{j}(b)\left(q_{j}(t)-q_{j}(t-T)\right)=0
\end{aligned}
$$

$\mu \ddot{V}_{D}+2 \xi_{D}\left[\dot{V}_{D}-\sum_{j} \phi_{j}(a) \dot{q}_{j}(t)\right]+\lambda_{D}^{4}\left[V_{D}-\sum_{j} \phi_{j}(a) q_{j}(t)\right]=0$

where

$\mu=\frac{M_{D}}{\rho A L}, \quad \xi_{D}=\frac{C_{D}}{2 \omega_{0} \rho A L}, \quad \lambda_{D}^{4}=\frac{K_{D}}{\omega_{0}^{2} \rho A L}, \quad k_{r}=\frac{k_{c} w_{c}}{\omega_{0}^{2} \rho A L}$,

$\omega_{k}=\lambda_{k}^{2} \omega_{0}, \quad \zeta_{k}=\frac{c}{2 \rho A \omega_{k}}$

Assuming that the dynamics of the beam is well represented by the first mode of vibration, we can reduce the system to the following two second-order equations that can be written in matrix form as

$$
\begin{aligned}
& \left(\begin{array}{ll}
1 & 0 \\
0 & \mu
\end{array}\right)\left\{\begin{array}{c}
\ddot{q}_{1}(t) \\
\ddot{V}_{D}(t)
\end{array}\right\}+\left(\begin{array}{cc}
2 \xi_{E} \lambda_{E}^{2} & -2 \xi_{D} \phi_{1}(a) \\
-2 \xi_{D} \phi_{1}(a) & 2 \xi_{D}
\end{array}\right)\left\{\begin{array}{c}
\dot{q}_{1}(t) \\
\dot{V}_{D}(t)
\end{array}\right\} \\
& +\left(\begin{array}{cc}
\lambda_{E}^{4}(1+Y) & -\lambda_{D}^{4} \phi_{1}(a) \\
-\lambda_{D}^{4} \phi_{1}(a) & \lambda_{D}^{4}
\end{array}\right)\left\{\begin{array}{c}
q_{1}(t) \\
V_{D}(t)
\end{array}\right\} \\
& +\left(\begin{array}{cc}
-\lambda_{E}^{4} Y & 0 \\
0 & 0
\end{array}\right)\left\{\begin{array}{c}
q_{1}(t-T) \\
V_{D}(t-T)
\end{array}\right\}=0
\end{aligned}
$$

with

$\lambda_{E}^{4}=\lambda_{1}^{4}+\lambda_{D}^{4} \phi_{1}^{2}(a), \quad \xi_{E}=\frac{\zeta_{1} \lambda_{1}^{2}+\xi_{D} \phi_{1}^{2}(a)}{\lambda_{E}^{2}}$

and

$Y=\frac{k_{r} \phi_{1}^{2}(b)}{\lambda_{E}^{4}}$

Solving the above equation system, the motion of the beam, using Eq. (4) restricted to the first mode $(N=1)$, and the motion of the DVA are obtained. A key point of this kind of problems is the analysis of the stability features dependent on operational conditions. This aspect is discussed in the next section.

\section{Stability analysis}

To analyze the stability of the solution of Eq. (12), we assume that

$q_{1}(t)=q_{10} e^{s t}, \quad V_{D}(t)=V_{D 0} e^{s t}$

where $q_{10}$ and $V_{D 0}$ are arbitrary constants and $s=\alpha+i \beta$ is the complex eigenvalue.

Substituting Eq. (15) in (12), we get a homogeneous system for the unknown constants $q_{10}$ and $V_{D 0}$. This system has nontrivial solutions if the following characteristic equation is satisfied:

$$
\left|\begin{array}{cc}
s^{2}+2 \xi_{E} \lambda_{E}^{2}+\lambda_{E}^{4}(1+Y)-\lambda_{E}^{4} Y e^{-s T} & -2 \xi_{D} \phi_{1}(a) s-\lambda_{D}^{4} \phi_{1}(a) \\
-2 \xi_{D} \phi_{1}(a) s-\lambda_{D}^{4} \phi_{1}(a) & \mu s^{2}+2 \xi_{D} s+\lambda_{D}^{4}
\end{array}\right|=0
$$

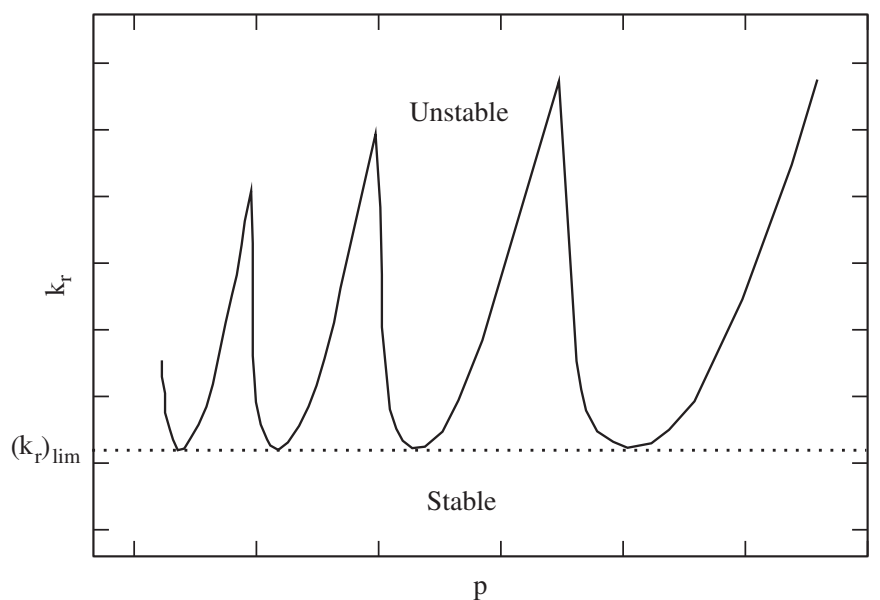

Fig. 2. Schematic view of the stability-lobes diagram.

The stability boundary is reached when purely imaginary eigenvalues occur, i.e. $s=i \beta$.

Now we introduce the auxiliary variables $W$ and $\tau$ given by

$W=\frac{\beta}{\lambda_{E}^{2}}, \quad \tau=\frac{T}{\lambda_{E}^{2}}$

Eq. (16) is equivalent to the two following equations corresponding to both real and imaginary part being null, respectively:

$$
\begin{aligned}
& \lambda_{E}^{4}\left(\left(\lambda_{D}^{4}-\mu \lambda_{E}^{4} W^{2}\right)\left(1-W^{2}+Y(1-\cos (W \tau))\right)+4 W^{2} \xi_{D}\left(\phi_{1}^{2}(a) \xi_{D}-\lambda_{E}^{2} \xi_{E}\right)\right) \\
& \quad-\phi_{1}^{2}(a) \lambda_{D}^{8}-2 W \lambda_{E}^{6} \xi_{D} \sin (W \tau)=0
\end{aligned}
$$

$$
\begin{aligned}
& W \lambda_{E}^{2} \xi_{D}\left(2 \lambda_{E}^{4}\left(1+Y-W^{2}\right)-4 \phi_{1}^{2}(a)\right)-2 W Y \lambda_{E}^{6} \xi_{D} \cos (W \tau) \\
& +\lambda_{E}^{4}\left(\lambda_{D}^{4}-W^{2} \lambda_{E}^{4} \mu\right)\left(2 W \xi_{E}+Y \sin (W \tau)\right)=0
\end{aligned}
$$

Thus, for each value of the auxiliary variable $W$ it is possible to solve Eqs. (18) and (19) obtaining the values of variables $Y$ and $\tau$ that satisfy Eq. (16) with the condition $s=i \beta$.

From the calculated values of $Y$ and $\tau$, we obtain

$k_{r}=\frac{k_{c} w_{c} L^{3}}{E I}=\frac{Y \lambda_{E}^{4}}{\phi_{1}^{2}(b)}$

$p=\frac{2 \pi \lambda_{E}^{2}}{\tau}=\frac{\Omega}{\omega_{0}}$

The curves $k_{r}$ versus $p$ constitute the stability boundaries of the system. Fig. 2 shows a schematic view of a typical stability-lobes diagram. The minimum values of these curves, $\left(k_{r}\right)_{\text {lim }}$, are related to the maximum value of chip width that can be removed irrespective to the turning speed. Then, for values of $k_{r}$ lower than $\left(k_{r}\right)_{\lim }$ the system is unconditionally stable.

\section{Absorber parameter selection with Den Hartog [41] and Sims [38] methods}

The vibration absorber should be designed from the standpoint of optimization of chatter stability. Two methodologies have been used for this objective: the classical tuning methodology of Den Hartog [41] and the recent work due to Sims [38]. Both methods analyze the effect of a mass attached to the undamped main structure (boring bar in this case) through an elastic spring and a viscous damper. From the analysis of the modulus of FRF, 
Den Hartog proposed a tuning frequency, as follows:

$f_{1}=\frac{\omega_{a}}{\omega_{1}}=\frac{1}{1+\mu^{*}}$

where $\omega_{a}$ is the absorber frequency given by $\omega_{a}=\sqrt{K_{D} / M_{D}}, \omega_{1}$ is the natural frequency of the main structure (in this case, $\omega_{1}$ corresponds to the fundamental frequency of the boring bar), and $\mu^{*}$ is the effective mass ratio given by

$\mu^{*}=\mu \phi_{1}^{2}(a)$

$\mu$ being the mass ratio written as $\mu=M_{D} / M_{S}$. Here, $M_{S}=\rho A L$ is the mass of the beam.

Note that the effective mass ratio must be considered in order to take into account the absorber position along the boring bar (see details in the Appendix).

Accordingly, Den Hartog [41] proposed a damping ratio as

$\xi_{1}=\sqrt{\frac{3 \mu^{*}}{8\left(1+\mu^{*}\right)}}$

In a recent work, Sims [38] proposed different values for the tuning frequencies and damping ratios, analyzing the real part of the FRF:

$f_{2}^{2}=\frac{\mu^{*}+2+\sqrt{2 \mu^{*}+\mu^{* 2}}}{2\left(1+\mu^{*}\right)^{2}}$

$f_{3}^{2}=\frac{\mu^{*}+2-\sqrt{2 \mu^{*}+\mu^{* 2}}}{2\left(1+\mu^{*}\right)^{2}}$

and

$\xi_{2}^{2}=\frac{\mu^{*}\left(\mu^{*}+3+\sqrt{2 \mu^{*}+\mu^{* 2}}\right)}{4\left(1+\mu^{*}\right)\left(\mu^{*}+2+\sqrt{2 \mu^{*}+\mu^{* 2}}\right)}$

$\xi_{3}^{2}=\frac{\mu^{*}\left(\mu^{*}+3-\sqrt{2 \mu^{*}+\mu^{* 2}}\right)}{4\left(1+\mu^{*}\right)\left(\mu^{*}+2-\sqrt{2 \mu^{*}+\mu^{* 2}}\right)}$

From a generic value of frequency $f$, given by Eqs. (22), (25) or (26) and $\xi$, given by Eqs. (24), (27) or (28), the corresponding dimensionless parameters characterizing the absorber used can be written as

$\lambda_{D}^{4}=\mu \lambda_{1}^{4} f^{2}$

$\xi_{D}=\xi \lambda_{D}^{2} \sqrt{\mu}=\xi \lambda_{1}^{2} \mu f$

and, finally, the dimensional stiffness and damping of the absorber are given by

$K_{D}=\lambda_{D}^{4} \frac{E I}{L^{3}}$

Table 1

Minimum values of stability-lobes diagram for different mass and positions of dynamic vibration absorber calculated with different methodologies.

\begin{tabular}{llllll}
\hline$\mu^{*}$ & \multicolumn{3}{l}{$\left(k_{r}\right)_{\text {lim }}$ obtained from } & & \\
\cline { 2 - 6 } & $f_{1}$ with $\xi_{1}$ & $f_{2}$ with $\xi_{2}$ & $f_{2}$ with $\xi_{3}$ & $f_{3}$ with $\xi_{2}$ & $f_{3}$ with $\xi_{3}$ \\
\hline 0.025 & 0.274 & 0.409 & 0.410 & - & - \\
0.050 & 0.397 & 0.603 & 0.605 & - & - \\
0.075 & 0.497 & 0.767 & 0.770 & - & - \\
0.100 & 0.583 & 0.909 & 0.913 & - & 0.413 \\
0.125 & 0.662 & 1.043 & 1.050 & 0.454 & 0.473 \\
0.150 & 0.732 & 1.163 & 1.173 & 0.502 & 0.525 \\
0.200 & 0.863 & 1.397 & 1.413 & 0.592 & 0.626 \\
0.250 & 0.981 & 1.611 & 1.634 & 0.672 & 0.716 \\
0.300 & 1.091 & 1.818 & 1.850 & 0.746 & 0.802 \\
0.400 & 1.290 & 2.205 & 2.258 & 0.878 & 0.959 \\
0.500 & 1.469 & 2.569 & 2.647 & 0.993 & 1.101 \\
\hline
\end{tabular}

Undamped boring bar.
$C_{D}=2 \xi_{D} \sqrt{\frac{\rho A E I}{L^{2}}}$

Then, given a mass ratio $\mu^{*}$ (obtained from the mass ratio $\mu$ and position $x_{a}\left(a=x_{a} / L\right)$ of the absorber, using Eq. (21)), five different combinations for tuning frequency and damping ratio can be selected. The combination of $f_{1}$ with $\xi_{1}$ corresponds to the analysis of Den Hartog [41] and from the Sims methodology [38] four cases can be analyzed corresponding to the use of the two possible optimal frequencies, $f_{2}$ and $f_{3}$ in conjunction with the two damping ratios, $\xi_{2}$ and $\xi_{3}$. The stability analysis was performed following the procedure explained in the previous section. The minimum value of the stability-lobes diagrams, $\left(k_{r}\right)_{\mathrm{lim}}$, corresponding to different values of $\mu^{*}$ and the five analyzed cases were resumed in Table 1 .

Based on the results shown in Table 1, it can be concluded that the best stability performance is achieved using the tuning frequency given by Eq. (25) ( $f_{2}$ of Sims model) combined with the damping ratio given by Eq. (28) ( $\xi_{3}$ of Sims model). The combination of frequency $f_{2}$ and the damping ratio $\xi_{2}$ of Sims model leads to similar results, but the minimum of the stabilitylobes diagram is slightly lower.

\section{Chatter stability improvement}

To investigate the existence of better values of the tuning frequency around that proposed by Sims [38], we performed an analysis varying the frequency in the proximity of the value given by Eq. (25), $f_{2}$, with a fixed damping ratio, because the tuning frequency plays a more significant role in the stability behavior than the damping ratio does.

From this local analysis it was possible to find a frequency denoted $f^{*}$, in the proximity of $f_{2}$, enhancing the stability behavior. Table 2 shows the values of the new frequency $f^{*}$ for different values of the parameter $\mu^{*}$. The frequency $f^{*}$ depends on the selected damping ratio (given by Eq. (27) or (28)). Thus the corresponding values of $\left(k_{r}\right)_{\text {lim }}$ are also dependent on this parameter. The use of damping ratio values given by Eq. (27) leads to higher values of $\left(k_{r}\right)_{\text {lim. }}$.

Fig. 3 shows the ratio between $f^{*} / f_{2}$ versus $\mu^{*}$ when the damping ratio is fixed to that given by Eq. (27) or (28). As can be seen, in both cases these relationships are approximately linear and the values have been fitted to straight lines whose approximate expressions are $f^{*} / f_{2}=\left(1+\mu^{*} / 2\right)$ and $f^{*} / f_{2}=\left(1+\mu^{*} / 4\right)$, respectively.

This result allowed us to propose new expressions for the optimal tuning frequency $f^{*}$ given by

$f_{1}^{*}=\left(1+\frac{\mu^{*}}{2}\right) \sqrt{\frac{\mu^{*}+2+\sqrt{2 \mu^{*}+\mu^{* 2}}}{2\left(1+\mu^{*}\right)^{2}}}$

Table 2

Optimum values for the tuning frequency.

\begin{tabular}{llllll}
\hline$\mu^{*}$ & \multicolumn{2}{l}{ Damping ratio $\xi_{2}$} & & \multicolumn{2}{l}{ Damping ratio $\xi_{3}$} \\
\cline { 2 - 3 } \cline { 5 - 6 } & $f^{*} / f_{2}$ & & & $f^{*} / f_{2}$ & $\left(k_{r}\right)_{\text {lim }}$ \\
& 1.007 & & & & \\
& 1.017 & 0.428 & & 1.005 & 0.423 \\
0.025 & 1.028 & 0.653 & & 1.011 & 0.636 \\
0.050 & 1.039 & 0.992 & & 1.018 & 0.825 \\
0.075 & 1.052 & 1.231 & & 1.031 & 0.992 \\
0.100 & 1.064 & 1.407 & & 1.037 & 1.155 \\
0.125 & 1.091 & 1.771 & & 1.048 & 1.033 \\
0.150 & 0.200 & & & & \\
\hline
\end{tabular}

Undamped boring bar. 


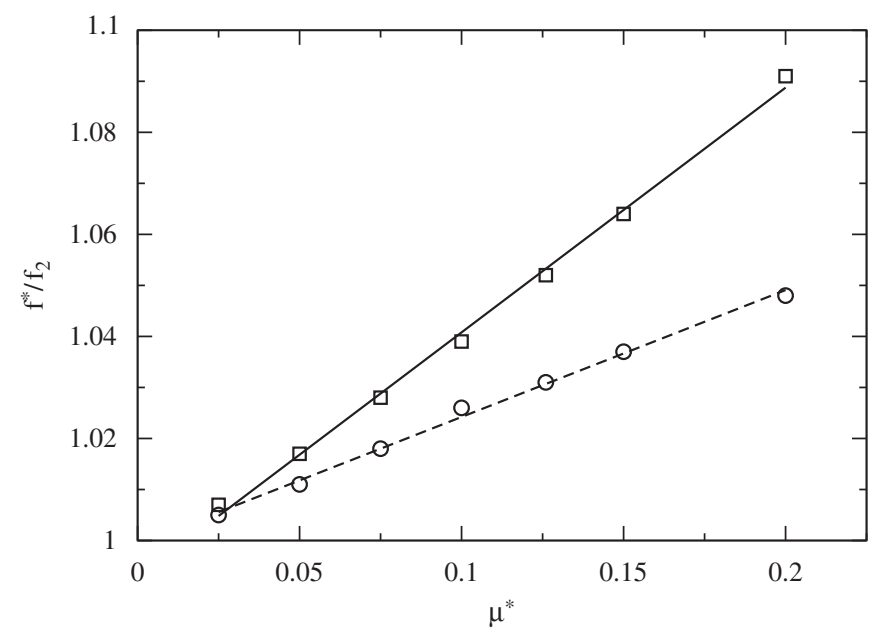

Fig. 3. Variation of optimum tuning frequency with the effective mass ratio of the vibration absorber. Undamped boring bar. ( $\square$ ) Damping ratio from Eq. (27). (०) Damping ratio from Eq. (28). (-) Fitted straight line equation (33). (- -) Fitted straight line equation (34).

Table 3

Minimum values of stability-lobes diagram for different mass and positions of dynamic vibration absorber calculated with different methodologies.

\begin{tabular}{llllll}
\hline$\mu^{*}$ & \multicolumn{5}{l}{$\left(k_{r}\right)_{\text {lim }}$ obtained from } \\
\cline { 2 - 6 } & $f_{1}$ with $\xi_{1}$ & $f_{2}$ with $\xi_{2}$ & $f_{2}$ with $\xi_{3}$ & $f_{3}$ with $\xi_{2}$ & $f_{3}$ with $\xi_{3}$ \\
\hline 0.025 & 0.571 & 0.679 & 0.682 & - & - \\
0.050 & 0.707 & 0.889 & 0.894 & - & - \\
0.075 & 0.819 & 1.067 & 1.075 & - & - \\
0.100 & 0.915 & 1.223 & 1.233 & - & 0.757 \\
0.125 & 1.004 & 1.369 & 1.383 & 0.804 & 0.823 \\
0.150 & 1.082 & 1.501 & 1.518 & 0.858 & 0.882 \\
0.200 & 1.229 & 1.755 & 1.780 & 0.959 & 0.994 \\
0.250 & 1.360 & 1.988 & 2.021 & 1.048 & 1.095 \\
0.300 & 1.482 & 2.213 & 2.256 & 1.131 & 1.190 \\
0.400 & 1.702 & 2.631 & 2.697 & 1.277 & 1.363 \\
0.500 & 1.898 & 3.023 & 3.117 & 1.405 & 1.519 \\
\hline
\end{tabular}

Damping ratio of the boring bar $\zeta_{1}=0.05$.

together with the damping ratio given by Eq. (27) or,

$f_{2}^{*}=\left(1+\frac{\mu^{*}}{4}\right) \sqrt{\frac{\mu^{*}+2+\sqrt{2 \mu^{*}+\mu^{* 2}}}{2\left(1+\mu^{*}\right)^{2}}}$

combined with the damping ratio given by Eq. (28).

These results were obtained for undamped main structure (boring bar). Note that the models of Den Hartog [41] and Sims [38] are strictly applicable only to this case. However, a similar analysis has been conducted with a damping ratio of the boring bar of $\zeta_{1}=0.05$. Similar trends were observed concerning the best frequency and damping ratio, although higher values of $\left(k_{r}\right)_{\lim }$ were found in this case, see Table 3.

Furthermore, an analysis varying the frequency in the proximity of the value obtained by Eq. (25), $f_{2}$, with a fixed damping ratio shows that, also in this case, it is possible to find a new frequency denoted $f_{\zeta}^{*}$, in the proximity of $f_{2}$, enhancing the stability behavior (see Table 4 ). Fig. 4 shows the ratio between $f_{\zeta}^{*} / f_{2}$ versus $\mu^{*}$ when the damping ratio is fixed to the given by Eq. (27) or (28). As can be seen, in both cases these relationships are approximately linear. The slopes of these straight lines are lower in comparison with those obtained with the undamped main structure.
Table 4

Optimum values for the tuning frequency.

\begin{tabular}{llllll}
\hline$\mu^{*}$ & \multicolumn{2}{l}{ Damping ratio $\xi_{2}$} & & \multicolumn{2}{l}{ Damping ratio $\xi_{3}$} \\
\cline { 2 - 3 } \cline { 5 - 6 } & $f_{\zeta}^{*} / f_{2}$ & & & $f_{\zeta}^{*} / f_{2}$ & $\left(k_{r}\right)_{\lim }$ \\
& 1.035 & & & \\
\hline 0.025 & 1.040 & 1.001 & & 1.035 & 0.764 \\
0.050 & 1.049 & 1.220 & & 1.042 & 0.996 \\
0.075 & 1.059 & 1.421 & & 1.049 & 1.202 \\
0.100 & 1.070 & 1.623 & & 1.055 & 1.388 \\
0.125 & 1.083 & 1.815 & & 1.061 & 1.734 \\
0.150 & 1.108 & 2.207 & & 1.061 & 2.057 \\
0.200 & 1.134 & 2.604 & 1.082 & 2.359 \\
0.250 & & & & & \\
\hline
\end{tabular}

Damping ratio of the boring bar $\zeta_{1}=0.05$.

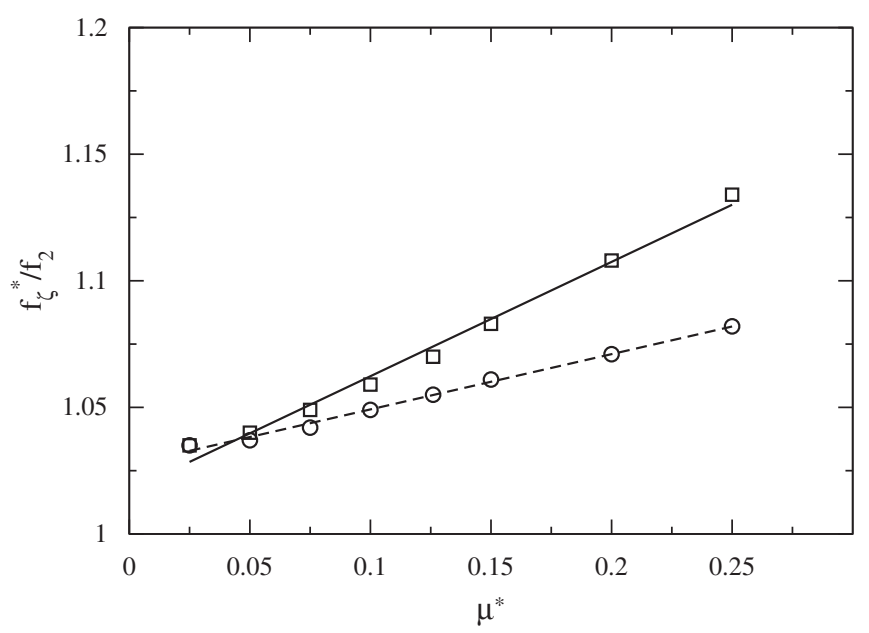

Fig. 4. Variation of optimum tuning frequency with the effective mass ratio of the vibration absorber. Damping ratio of the boring bar, $\xi=0.05$. ( $\square$ ) Damping ratio from Eq. (27). ( $)$ Damping ratio from Eq. (28). (-) Fitted straight line. (- -) Fitted straight line.

Table 5

Parameters of the boring bar and mass and position of the dynamic absorber.

\begin{tabular}{llll}
\hline Boring bar (main structure) & Dynamic vibration absorber \\
\hline Length, $L$ & $0.30 \mathrm{~m}$ & Mass, $M_{D}$ & $0.05 \mathrm{~kg}$ \\
Diameter, $D$ & $0.02 \mathrm{~m}$ & Distance to clamping, $x_{a}$ & $0.195 \mathrm{~m}$ \\
Section, $A$ & $3.142 \times 10^{-4} \mathrm{~m}^{2}$ & $a=x_{a} / L$ & 0.65 \\
Inertia, $I$ & $7.854 \times 10^{-9} \mathrm{~m}^{4}$ & Mass ratio, $\mu$ & 0.068 \\
Density, $\rho$ & $7850 \mathrm{~kg} / \mathrm{m}^{3}$ & Effective mass ratio, $\mu^{*}$ & 0.075 \\
Mass of the beam, $M_{S}$ & $0.735 \mathrm{~kg}$ & & \\
Young modulus, $E$ & $2 \times 10^{11} \mathrm{~Pa}$ & & \\
Cutting force position, $x_{b}$ & $0.294 \mathrm{~m}$ & & \\
$b=x_{b} / L$ & 0.98 & & \\
\hline
\end{tabular}

\section{Practical example}

To illustrate the methodology proposed in the paper, a practical example concerning a real boring bar is presented in this section. Table 5 summarizes boring bar characteristics being common values used in industrial tools. Thus, a steel boring bar whit length $L=0.30 \mathrm{~m}$ and a circular cross-section of diameter $D=0.02 \mathrm{~m}$ is considered. The mass of the bar is $M_{S}=0.735 \mathrm{~kg}$ and the length-to-diameter ratio of the boring bar $(L / D)$ is 15 . The dynamic absorber has a mass of $M_{D}=0.05 \mathrm{~kg}$ and is located at a distance from the clamping end of $a=0.195 \mathrm{~m}$. It is important to note that both the mass and the position of the absorber along the 


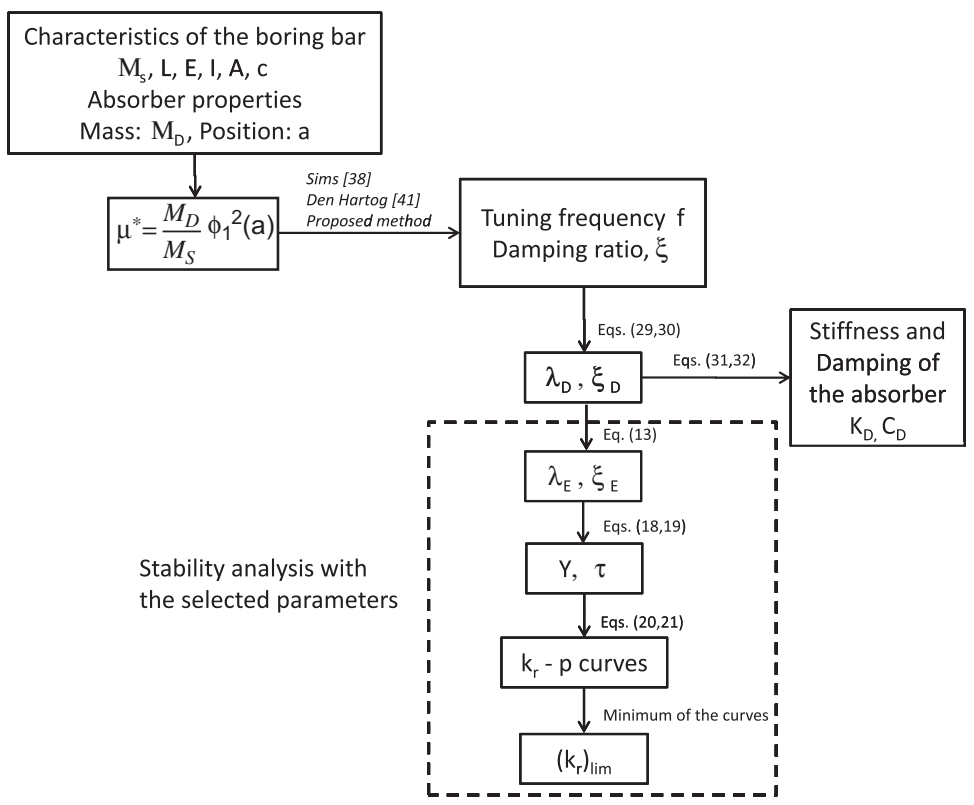

Fig. 5. Calculation scheme for parameter selection and stability analysis.

Table 6

Stiffness and damping of the dynamic absorber selected with different methodologies.

\begin{tabular}{|c|c|c|c|c|c|c|c|c|}
\hline Method & & $f$ & $\xi$ & $\lambda_{D}$ & $\xi_{D}$ & $\left(k_{r}\right)_{\lim }$ & $K_{D}(\mathrm{~N} / \mathrm{m})$ & $C_{D}(\mathrm{~N} \mathrm{~m} / \mathrm{s})$ \\
\hline Den Hartog & $\left(f_{1}\right.$ with $\left.\xi_{1}\right)$ & 0.930 & 0.162 & 0.924 & 0.036 & 0.496 & 42326 & 14.88 \\
\hline Sims 1 & $\left(f_{2}\right.$ with $\left.\xi_{2}\right)$ & 1.034 & 0.157 & 0.974 & 0.039 & 0.766 & 52265 & 16.00 \\
\hline Sims 2 & $\left(f_{2}\right.$ with $\left.\xi_{3}\right)$ & 1.034 & 0.167 & 0.974 & 0.041 & 0.768 & 52265 & 17.06 \\
\hline Proposed 1 & $\left(f_{1}^{*}\right.$ with $\left.\xi_{2}\right)$ & 1.072 & 0.157 & 0.992 & 0.040 & 0.817 & 56261 & 16.61 \\
\hline Proposed 2 & $\left(f_{2} *\right.$ with $\left.\xi_{3}\right)$ & 1.053 & 0.167 & 0.983 & 0.042 & 0.820 & 54245 & 17.38 \\
\hline
\end{tabular}

bar should not interfere with its ability to perform the boring operation. Finally we suppose the cutting force is applied near the free end of the boring bar $(b=0.294 \mathrm{~m})$.

In order to select the other characteristics of the dynamic absorber, the calculation scheme presented in the foregoing section is showed in Fig. 5 and applied for the data given in Table 5. The results were compared to those obtained using Den Hartog and Sims methodologies, and the main results of the comparative analysis are given in Table 6 . The optimal solution is found for the stiffness of the absorber, $K_{D}=56.3 \mathrm{~N} / \mathrm{mm}$, and damping, $C_{D}=16.6 \mathrm{~N} \mathrm{~s} / \mathrm{m}$, that are in the range proposed by other authors (see [38], for instance).

Note that the values given in Table 6 are slightly different to those shown in Table 2 for the same value of $\mu^{*}(0.075)$. These differences are due to the use of approximate values of $f^{*}$ in the example, given by Eqs. (33) and (34), instead of those obtained in the local analysis.

In any case, improvement of stability behavior was obtained with the procedure proposed in this work. The minimum of the stability diagram (being the upper limit of unconditional stability zone) was increased when compared with the values given by Den Hartog and Sims methodologies. In fact the increments were $64 \%$ and $7 \%$, respectively.

As it has been previously stated, the model does not consider the effect of insert nose radius. Ozlu and Budak [26] conducted boring experiments with different insert nose radii and they found that the use of inserts with an increasing nose radius reduces the stability limit. In boring operations the tool is clamped in such a way that it is much more flexible than the workpiece, being this flexibility the common problem in current boring operations. The change in the absolute stability limit is due to the sudden increase of the effect of the flexible tool on the rigid dynamic system.

Although in this work the effect of nose radius has not been analyzed, the same trends observed by Ozlu and Budak [26] for the case without any passive absorber could be expected.

On the other hand, the methodology presented in the paper properly solve the problem of the stability of the boring bar with a passive absorber subjected to the classical force system considered in regenerative chatter process, improving the well established method previously proposed by Sims [38], but it could be applied in more general situations, including, for instance, the effect of nose radius in the dynamics of the system and subsequently in the stability limit.

\section{Concluding remarks}

This work is focused on the design improvement of passive dynamic absorbers (DVA) for chatter suppression in boring operations. The boring bar, being the main structure, was modeled as a Euler-Bernoulli beam, accounting only the first vibration mode. The absorber parameters (mass, stiffness, damping, and position) were considered to construct the stability-lobes diagram. Two analytical approaches proposed by Den Hartog [41] and Sims [38] were used to determine the optimum parameters of the absorber. The selection criterion was to maximize the minimum values of the stability-lobes diagram. It was demon- 
strated that the approach presented by Sims led to a wider stability zone than that proposed by Den Hartog. Moreover, subsequent local analysis performed in this work, allowed the establishment of simple analytical expressions for the tuning frequency improving the behavior of the system against chatter. The method could be easily implemented in the design procedure of passive absorbers in boring operations. The procedure was successfully applied in a practical example illustrative of a real case of boring operation in industry.

\section{Appendix A}

The motion equations of the two-degree-of-freedom system representing the boring bar with and attached damper can be written as

$[\mathbf{M}]\{\ddot{\mathbf{X}}\}+[\mathbf{C}]\{\dot{\mathbf{X}}\}+[\mathbf{K}]\{\mathbf{X}\}=\{\mathbf{F}\}$

with

$[\mathbf{M}]=\left(\begin{array}{cc}M_{S} & 0 \\ 0 & M_{D}\end{array}\right), \quad[\mathbf{C}]=\left(\begin{array}{cc}C_{D} \phi_{1}^{2}(a) & -C_{D} \phi_{1}(a) \\ -C_{D} \phi_{1}(a) & C_{D}\end{array}\right)$

$[\mathbf{K}]=\left(\begin{array}{cc}K+K_{D} \phi_{1}^{2}(a) & -K_{D} \phi_{1}(a) \\ -K_{D} \phi_{1}(a) & K_{D}\end{array}\right), \quad\{\mathbf{F}\}=\left(\begin{array}{c}F_{0} \\ 0\end{array}\right)$

By assuming the solution to be

$x_{j}=X_{j} e^{i \omega t}, \quad j=1,2$

With the following definitions:

$\mu=\frac{M_{D}}{M_{S}}, \quad f=\frac{\omega_{D}}{\omega_{1}}, \quad \delta_{s t}=\frac{F_{0}}{K}$

$\omega_{D}=\sqrt{\frac{K_{D}}{M_{D}}}, \quad \omega_{1}=\sqrt{\frac{K}{M}}$

$\xi=\frac{C_{D}}{2 M_{D} \omega_{D}}, \quad g=\frac{\omega}{\omega_{1}}, \quad \mu^{*}=\mu \phi_{1}^{2}(a)$

the steady state-solution for the motion of main structure can be obtained as

$\frac{X_{1}}{\delta_{s t}}=\frac{\left(f^{2}-g^{2}\right)+i 2 \xi g f}{\left(1-g^{2}\right)\left(f^{2}-g^{2}\right)-\mu^{*} f^{2} g^{2}+i 2 \xi f g\left(1-g^{2}-\mu^{*} g^{2}\right)}$

Note that the last equation coincide with the well-known expression derived for a system with two degrees of freedom (see for instance [38]), just substituting the mass ratio $\mu$ by the effective mass ratio $\mu^{*}=\mu \phi_{1}^{2}(a)$.

\section{References}

[1] Altintas Y. Manufacturing automation: metal cutting, mechanics, machine tool vibrations, and CNC design. Cambridge, UK: Cambridge University Press; 2000.

[2] Taylor FW. On the art of cutting metals. Transactions of the ASME 1907;28:31-350.

[3] Arnold RN. The mechanism of tool vibration in cutting of steel. Proceedings of the Institution of Mechanical Engineers 1946;154:261-84.

[4] Tobias SA, Fishwick W. Theory of regenerative machine tool chatter Engineering 1958;205:16-23.

[5] Tlusty J, Polacek M. The stability of machine tools against selfexcited vibrations in machining. International Research in Production Engineering, ASME 1963:465-74.

[6] Merritt HE. Theory of self-excited machine-tool chatter, contribution to machine-tool chatter, research 1 . Journal of Engineering for Industry, Transactions of the ASME 1965;87:447-54.

[7] Tobias SA. Machine-tool vibration. London: Blackie and Son Limited; 1965

[8] Hanna NH, Tobias SA. A theory of nonlinear regenerative chatter. Journal of Engineering for Industry-Transactions of the ASME 1974;96:247-55.

[9] Wu D, Liu C. An analytical model of cutting dynamics. Part 1: model building. Journal of Engineering for Industry-Transactions of the ASME 1985;107: 107-11.
[10] Wu D, Liu C. An analytical model of cutting dynamics. Part 2: verification. Journal of Engineering for Industry-Transactions of the ASME 1985; $107: 112-8$

[11] Budak E, Altintas Y. Analytical prediction of chatter stability in milling-part I: general formulation. Journal of Dynamic Systems, Measurement, and Control 1998;120:22-30.

[12] Budak E, Altintas Y. Analytical prediction of chatter stability in milling-part II: application of the general formulation to common milling systems. Journal of Dynamic Systems, Measurement, and Control 1998;120: $31-6$.

[13] Insperger T, Stepan G. Stability of high speed milling. In: Proceedings of the 2000 ASME international mechanical engineering congress and exposition, Orlando, Florida, 2000, AMD-241. p. 119-23.

[14] Bayly PV, Mann BP, Schmitz TL, Peters DA, Stepan G, Insperger T. Effects of radial immersion and cutting direction on chatter instability in end-milling. In: Proceedings of the 2002 ASME international mechanical engineering congress and exposition, New Orleans, Louisiana, 2002, paper no. IMECE2002-34116 (CD-ROM).

[15] Sims ND, Manson G, Mann B. Fuzzy stability analysis of regenerative chatter in milling. Journal of Sound and Vibration 2010;329:1025-41.

[16] Vela-Martinez L, Jauregui-Correa J, Rubio-Cerda E, Herrera-Ruiz G, Lozano Guzman A. Analysis of compliance between the cutting tool and the workpiece on the stability of a turning process. International Journal of Machine Tools and Manufacture 2008;48:1054-62.

[17] Parker EW. Dynamic stability of a cantilever boring bar with machined flats under regenerative cutting conditions. Journal of Mechanical Engineering Science 1970;12:104-15.

[18] Andren L, Hakansson L, Brandt A, Claesson I. Identification of dynamic properties of boring bar vibrations in a continuous boring operation. Mechanical Systems and Signal Processing 2004;18:869-901.

[19] Akesson H, Smirnova T, Hakansson L. Analysis of dynamic properties of boring bars concerning different clamping conditions. Mechanical Systems and Signal Processing 2009;23:2629-47.

[20] Zhang GM, Kapoor SG. Dynamic modeling and analysis of the boring machining system. Journal of Engineering for Industry-Transactions of the ASME 1987;109:219-26.

[21] Andren L, Hakansson L, Brandt A, Claesson I. Identification of motion of cutting tool vibration in a continuous boring operation-correlation to structural properties. Mechanical Systems and Signal Processing 2004; 18:903-27.

[22] Rao PN, Rao URK, Rao JS. Towards improved design of boring bars. I: dynamic cutting force model with continuous system-analysis for the boring bar performance. International Journal of Machine Tools and Manufacture 1988;28:33-44.

[23] Kuster F, Gygax P. Cutting dynamics and stability of boring bars. CIRP Annals-Manufacturing Technology 1990;39:361-6.

[24] Lazoglu I, Atabey F, Altintas Y. Dynamics of boring processes: part III-time domain modeling. International Journal of Machine Tools Manufacture 2006;42:1567-76.

[25] Ozlu E, Budak E. Analytical modeling of chatter stability in turning and boring operations-part I: model development. Journal of Manufacturing Science and Engineering 2007;129:726-32.

[26] Ozlu E, Budak E. Analytical modeling of chatter stability in turning and boring operation-part II: experimental verification. Journal of Manufacturing Science and Engineering 2007;129:733-9.

[27] Moetakef-Imani B, Yussefian NZ. Dynamic simulation of boring process. International Journal of Machine Tools Manufacture 2009;49: 1096-103.

[28] Wang M, Fei R. Chatter suppression based on nonlinear vibration characteristic of electrorheological fluids. International Journal of Machine Tools and Manufacture 1999;39:1925-34.

[29] Mei D, Kong T, Shih AJ, Chen Z. Magnetorheological fluid-controlled boring bar for chatter suppression. Journal of Materials Processing Technology 2009;209:1861-70.

[30] Tewani S, Rouch K, Walcott B. A study of cutting process stability of a boring bar with active dynamic absorber. International Journal of Machine Tools and Manufacture 1995;35:91-108.

[31] Pratt JR, Nayfeh AH. Chatter control and stability analysis of a cantilever boring bar under regenerative cutting conditions. Philosophical Transactions of the Royal Society A: Mathematical, Physical and Engineering Sciences 2001;359:759-92.

[32] Mei C. Active regenerative chatter suppression during boring manufacturing process. Robotics and Computer-Integrated Manufacturing 2005;21: $153-8$.

[33] Akesson H, Smirnova T, Claesson I, Hakansson L. On the development of a simple and robust active control system for boring bar vibration in industry. International Journal of Acoustics and Vibration 2007;12:139-52.

[34] Rivin EI, Kang H. Enhancement of dynamic stability of cantilever tooling structures. International Journal of Machine Tools and Manufacture 1992:32:539-61.

[35] Tarng YS, Kao JY, Lee EC. Chatter suppression in turning operations with a tuned vibration absorber. Journal of Materials Processing Technology 2000;105:55-60

[36] Ema S, Marui E. Suppression of chatter vibration of boring tools using impact dampers. International Journal of Machine Tools and Manufacture 2000;40:1141-56 
[37] Lee E, Nian C, Tarng Y. Design of a dynamic vibration absorber against vibrations in turning operations. Journal of Materials Processing and Technology 2001;108:278-85

[38] Sims ND. Vibration absorbers for chatter suppression: a new analytical tuning methodology. Journal of Sound and Vibration 2007;301: 592-607.
[39] Moradi H, Bakhtiari-Nejad F, Movahhedy M. Tuneable vibration absorber design to suppress vibrations: an application in boring manufacturing process. Journal of Sound and Vibration 2008;318:93-108.

[40] Saffury J, Altus E. Optimized chatter resistance of viscoelastic turning bars. Journal of Sound and Vibration 2009;324:26-39.

[41] Den Hartog JP. Mechanical vibrations. fourth ed. New York: McGraw-Hill; 1956. 